\title{
Redesign and Evaluation of AffectiVest, a Wearable Tool to Convey Affection Gestures between Geographically-Separated Grandparents and Grandchildren ${ }^{\dagger}$
}

\author{
Flor B. Montañez ${ }^{1, \ddagger, \S \subseteq}$, Victoria Meza-Kubo ${ }^{1, \S}$, Cristina Ramírez-Fernández ${ }^{2, \S}$ and \\ Alberto L. Morán 1,*,§ \\ 1 Facultad de Ciencias, Universidad Autónoma de Baja California, Ensenada, 22860, México; \\ flor.montaez@uabc.edu.mx (F.B.M.); mmeza@uabc.edu.mx (V.M.-K.) \\ 2 División de Estudios de Posgrado e Investigación, Tecnológico Nacional de México/I.T. de Ensenada, \\ Ensenada, 22780, México; cramirez@ite.edu.mx \\ * Correspondence: alberto.moran@uabc.edu.mx; Tel.: +52-646-174-4560 \\ + Presented at the 13th International Conference on Ubiquitous Computing and Ambient Intelligence \\ UCAmI 2019, Toledo, Spain, 2-5 December 2019. \\ $\ddagger$ Current address: km.103 carretera Tijuana-Ensenada, México. \\ $\S$ These authors contributed equally to this work.
}

Published: 21 November 2019

\begin{abstract}
Motivated by the need to improve communication between grandparents and grandchildren who are geographically separated, this paper presents the redesign and evaluation of AffectiVest, a wearable affective device that allows the transmission of affection gestures between grandparents and grandchildren. The redesign consisted of modifying the architecture of AffectiVest to allow wireless connectivity and mobility, and modifying the implementation of the stimuli that represent the affection gestures to make them more similar to the real ones. AffectiVest was evaluated by 10 grandparent-grandchild pairs who interacted using the device for several minutes, actually wearing the device while communicating in a separated environment. The results of the Software Usability Scale (SUS) and Technology Acceptance Model (TAM) on-exit questionnaires, as well as the questionnaire of fidelity of the affection gestures, allowed us to establish that the redesign of the device was successful, since it was perceived by its users as having a high usability, and as being adequate and intuitive at evoking all the affection gestures as real enough. In addition, it allowed identifying a set of specific recommendations from the group of target users regarding the adjustment of the vest and the location of the actuators considering the differences in the size of the users, and the possibility of using the vest while carrying out the activities of daily living.
\end{abstract}

Keywords: affective wearable computing; transmission of affection gestures; grandparent-grandchild relation

\section{Introduction}

Current estimates are that there are 244 million international migrants globally (or 3.3\% of the world's population) [1]. One of the highest costs that migrants and their families have to face is family separation that particularly affects the relationship between grandparents and grandchildren. In the literature there is evidence of the benefits of the grandparent-grandchild relationship; according to studies carried out in $[2,3]$ it is established that this relationship is substantial for both of them and that it is considered as the second most important link in comparison with that of parent and child. In addition, in these studies it is argued that grandparents are considered in the family as 
caregivers and responsible for reinforcing family history, providing them with joy and a meaning of life avoiding social isolation; while for grandchildren having a close relationship with their grandparents gives them emotional security by providing an almost unconditional love. Continuous contact with grandparents and parental intervention help building strong relationships over time [4,5]. When there is geographic separation, the grandparents establish communication with their grandchildren through different electronic means, seeking to communicate affection to the extent that they are allowed by the used media p. ex. kisses and hugs through gestures, sounds and changes in the tone of voice. However, these means of communication usually are visual and/or auditory, which makes it difficult for grandparents to maintain the attention of small grandchildren [6]. There are other factors that may affect the relationship such as social circumstances, time zones, cultural differences or language barriers [7]. Motivated by the need to transmit affection gestures technologies have been proposed that allow us to transmit emotions and provide stimuli with affective meanings for users; one technology that meets these characteristics is Affective Wearable Computing (AWC). Affective wearable computing is defined as a wearable system equipped with sensors and instruments that allow the recognition, transmission and interpretation of affective patterns of the wearer [8]. The development of AWC devices would make possible to reproduce the feelings of affection in real time as real as possible, and facilitate maintaining a closer and more constant relationship between loved ones geographically separated [9,10]. This paper presents the redesign of AffectiVest, an AWC device whose purpose is to transmit affection gestures between geographically separated grandparents and grandchildren to enrich their communication processes and strengthen their affective ties. In addition, the results of a preliminary evaluation of usability, usefulness and ease of use of couples of the target group of users (grandparents and grandchildren), are presented.

\section{Related Work}

In the AWC literature there are devices whose functionality focuses on transmitting affection gestures between couples sending signals from a mobile/desktop application that the user interprets as emotions and that are displayed on a wearable device. For example, Hug Over a Distance [9], is a device created for couples geographically separated with the aim of proving the viability of the technology from the privacy perspective. This device is designed as a haptic vest that inflates itself to provide the feeling of receiving a hug. Couples who used the device in the evaluation did not consider the vest useful in their daily life; however, the developers proposed that the device can be useful to obtain design ideas in the research. Another device for commercial use aimed at sending hugs between couples is The Hug Shirt [11], which incorporates sensors and actuators to simulate a hug based on tactile pressure, heartbeat and temperature change. Another device to simulate hugs is Huggy Pajama [10], which helps fostering the relationship between parents and their children using color change patterns to indicate the distance of separation between users and provide pressure and temperature tactile stimuli to generate the hug. There is also a device developed for children and older adults who need to spend a lot of time in a hospital receiving therapies, called Affective tele-touch [12], which through a haptic device placed on the forearm of the user transmits an affective touch generated by vibrations and temperature change; its evaluation results show, in terms of the user's heart rate, that there was no significant difference between receiving a touch with the device and from a loved one. The aforementioned devices focus on reproducing a single affection gesture using touch as a form of communication; however, very few of these devices have been evaluated in practice or do not present information of having carried out an evaluation that allows us to determine the fidelity of the affection gestures and/or the user perceived usability regarding the use of the device to send affection gestures between grandparents and grandchildren. 


\section{Previous Work}

In this section, we provide a brief description of the design of an initial version of AffectiVest, along with the results of a preliminary evaluation of the fidelity of its affection gestures as perceived by young adults (reported elsewhere [13]).

\subsection{Device Description}

The initial version of AffectiVest was designed to transmit and evoke affection gestures between grandparents and grandchildren who are geographically separated, aiming at stimulating the feeling of closeness and family bonding. In its basic operation, the device is used between two geographically separated users, a sender and a receiver. Firstly, the sender was simulated with a desktop application that allowed selecting the affection gestures to be used, and it had a wearable client application that reproduced the affection gestures sent from the sender application. This version of the AffectiVest prototype implements four affection gestures: hugging, kissing, tickling in the abdomen and tapping on the shoulder. For its operation, it is necessary to establish a wired connection between them to allow communication. In its first implementation, the wearable device was developed as a vest, with the intention of placing it over the user's clothing while being able to adjust it with velcro to fit to different user sizes (see Figure 1). To reproduce the hug a bag that was inflated using an air pump was used, allowing to exert a pressure on the chest of the user simulating that s/he had received a hug. For the representation of the kiss we used the audio of a kiss that was reproduced by means of a speaker placed at the height of the right shoulder of the wearable device. To represent the tap on the shoulder, a sequence of two vibrations was applied on the left shoulder through a mini vibrating motor. Finally, to represent the tickle, a sequence of vibrations was used using two mini vibrator motors placed at the height of the abdomen. These components were controlled by means of an Arduino UNO, which was programmed to activate each of these affection gestures.

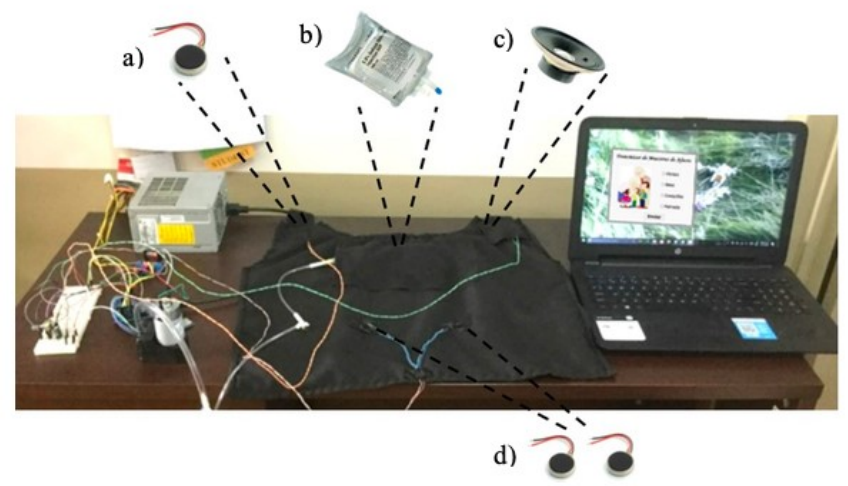

Figure 1. First AffectiVest prototype; (a) tap representation; (b) hug representation; (c) kiss representation; (d) tickle representation.

\subsection{Preliminary Evaluation}

A preliminary evaluation was conducted using this version of the AffectiVest AWC prototype with the objective of identifying if it was possible to evoke affection gestures through the stimuli provided by the prototype and to know the fidelity with which they were perceived by the participants. The experiment involved 33 undergraduate students from different programs of a local university; their average age was 20.5 years (SD 1.78), 20 were men and 13 women. The experiment results showed that the hugging and kissing affection gestures evoked such affection gestures 57.57\% (57/99) and $53.53 \%$ (53/99) of the times, respectively; while the tickling and tapping gestures evoked such affection gestures 19.19\% (19/99) and 3.03\% (3/99) of the time, respectively. Although hugging was the most well evoked gesture, several participants mentioned that the air pump used to inflate the bag was too noisy and that it did not correspond to an actual hugging affection gesture. It was concluded 
from this evaluation that it is possible to transmit affection gestures using the proposed AffectiVest prototype to be used by people who are geographically separated, but it is necessary to redesign the tickling and tapping on the shoulder affection gestures so that they resemble more to the real ones, and the hugging affection gesture in order to eliminate the noise produced by the air pump. Additionally, the implementation of the vest must be redesigned using wireless technology to provide a more comfortable service to participants.

\section{Redesign of the AffectiVest Prototype}

\subsection{Redesign of the Architecture}

A first modification of the architecture of the AffectiVest system was the use of a Bluetooth connection between the sender application and the receiver application of the wearable device, eliminating the wired connection of the previous version. Figure 2 illustrates the current architecture of the prototype, depicting the various components of the sender and receiver applications, the smartphone and the vest showing the location of the actuators used to implement the four affection gestures.
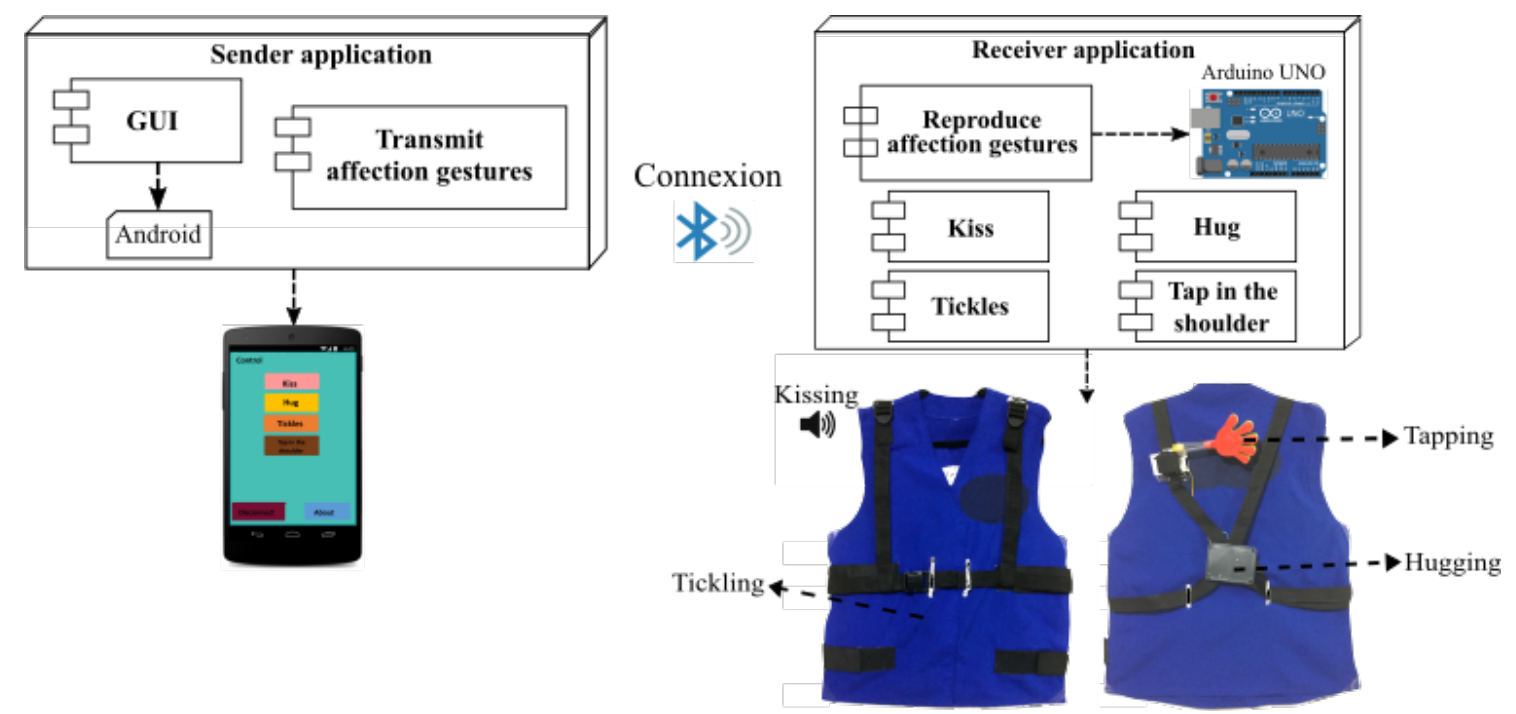

Figure 2. Current architecture of the AffectiVest Affective Wearable Computing (AWC) prototype.

\subsection{Redesign of the Sender Application}

The sender application was modified, going from a desktop application to a mobile application on a smartphone with Android operating system. The starting procedure of the mobile App is as follows. First the user has to search and select the icon of the sender application on the smartphone, then select the wearable device to synchronize it with the sender application to establish connection (see Figure 3a). After the connection verification message, an entry screen appears for a few seconds. Finally the GUI of the sender application appears (see Figure 3b), composed of four buttons (one for each affection gesture) and two more buttons, one to disconnect from the wearable device and the other to see information about the developers. 


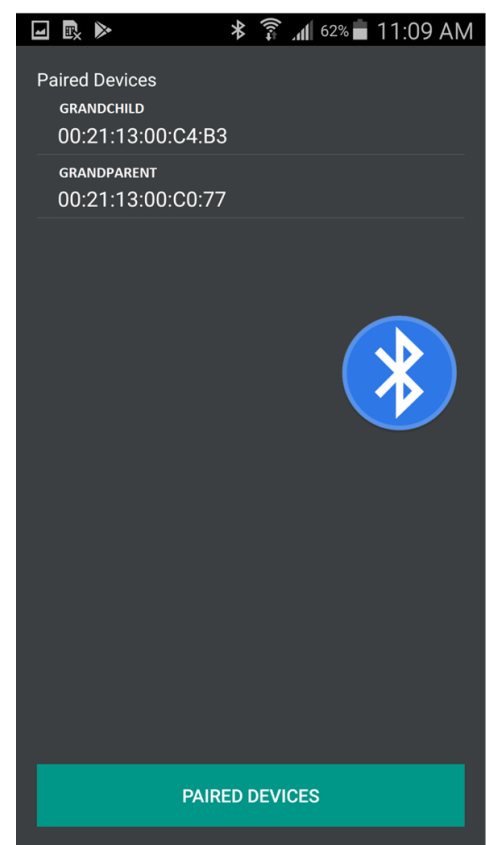

(a)

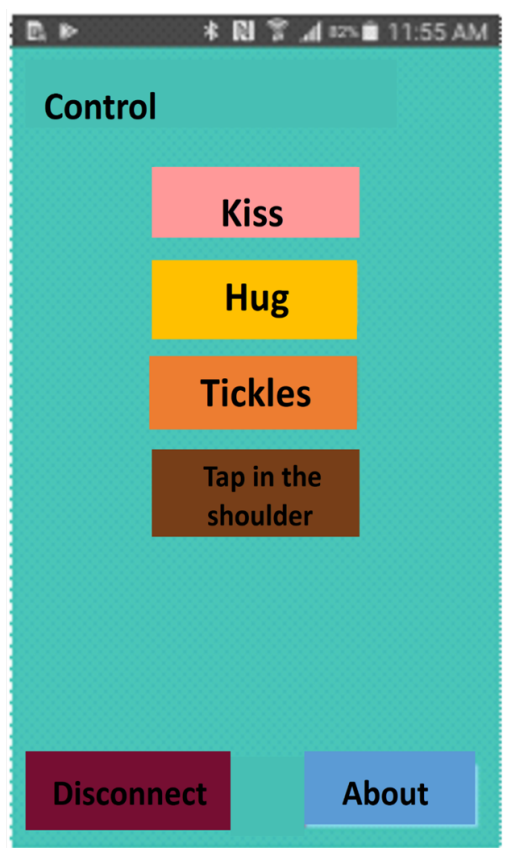

(b)

Figure 3. Screenshots of the sender application. (a) synchronization between sender and receiver applications and (b) GUI of the sender application to send the affection gestures.

\subsection{Redesign of the AWC Device}

The appearance of the wearable device was slightly modified, respecting the criterion of being a vest that could be placed over the clothes of the users and adapted to the different sizes. Like the first prototype, this redesigned version contains different actuators that are controlled by an Arduino UNO board to reproduce the four affection gestures that are sent from the sender application.

As mentioned before, based on the results presented in the previous work [13], it was proposed to redesign the implementation of the affection samples "tickling in the abdomen" and "tapping on the shoulder", which were less perceived and/or evoked as real by users. The "hugging" gesture was also redesigned, given that users suggested that the vacuum pump used to inflate the bag in the previous version caused a lot of noise when turned on, which distracted the participants in some cases.

\subsubsection{Hugging Affective Gesture}

To eliminate the bag and air pump from the representation of this affection gesture, a strap was used on the wearable device, fitting around the wearer's torso. The strap is wound by means of a gear motor that was responsible for tightening one end of the strap and exerting a slight pressure around the torso of the user, with the intention of generating the sensation of "embracing with the arms" and pretending to have received a hug (see Figure 4).

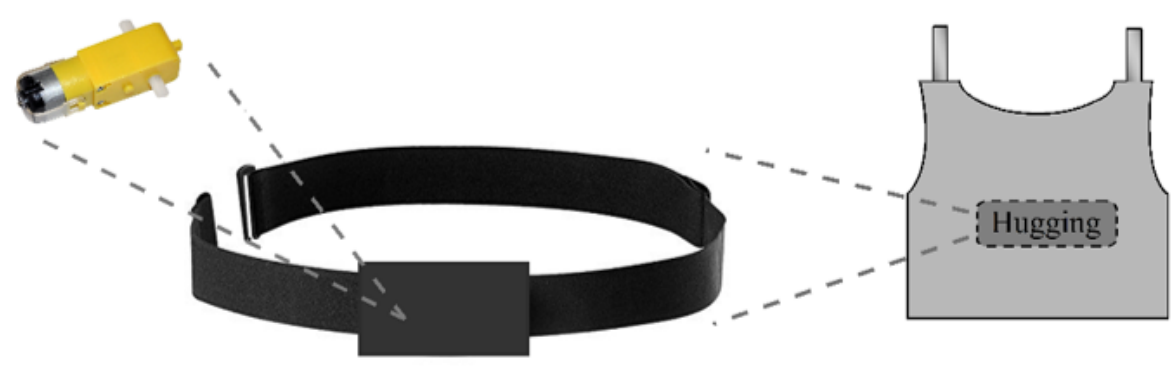

Figure 4. Location of the strap and actuator for the hugging affection gesture. 


\subsubsection{Tickling in the Abdomen Affection Gesture}

As in the previous version of AffectiVest, the tickling in the abdomen affection gesture was represented using vibrations. However, in this version six commercial mini vibrator motors were used, which were placed in the wearable device at the height of the user's abdomen, causing the receiving user to feel tickle in the abdomen (see Figure 5).

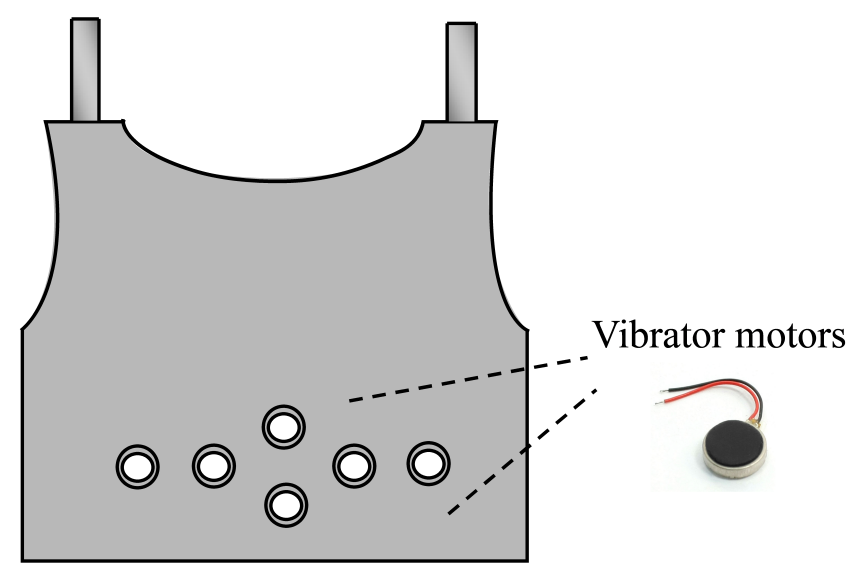

Figure 5. Location of the actuators for the tickling in the abdomen.

\subsubsection{Tapping on the Shoulder Affection Gesture}

This affection gesture was represented using a plastic object with the appearance of a human hand at smaller scale, which was attached to a servomotor and placed on the back of the AffectiVest. For the movement of the hand an HS 422 servomotor was used, which is configured to perform up and down movements with 2 degrees of freedom and with an angle of 45 degrees; causing gentle hits on the shoulder of the wearer of the vest to provide the feeling of having received shoulder tappings (see Figure 6).

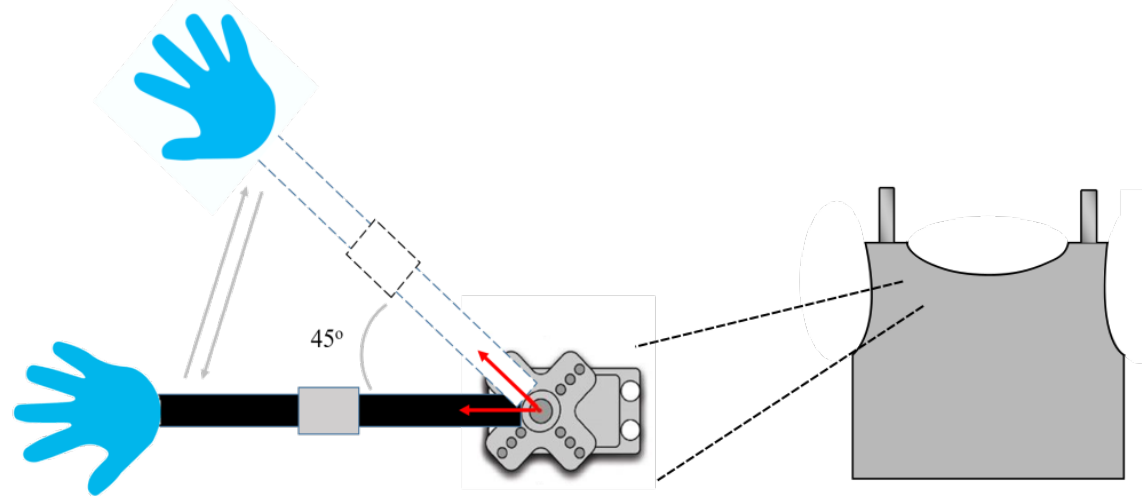

Figure 6. Location of the actuator for the tapping on the shoulder affection gesture.

\subsubsection{Kissing Affection Gesture}

As in the previous version, for the representation of the kissing affection gesture, an 8 ohm speaker was used located on the vest at the height of the wearer's shoulder, which reproduced the sound of a pre-recorded kiss (see Figure 7). 


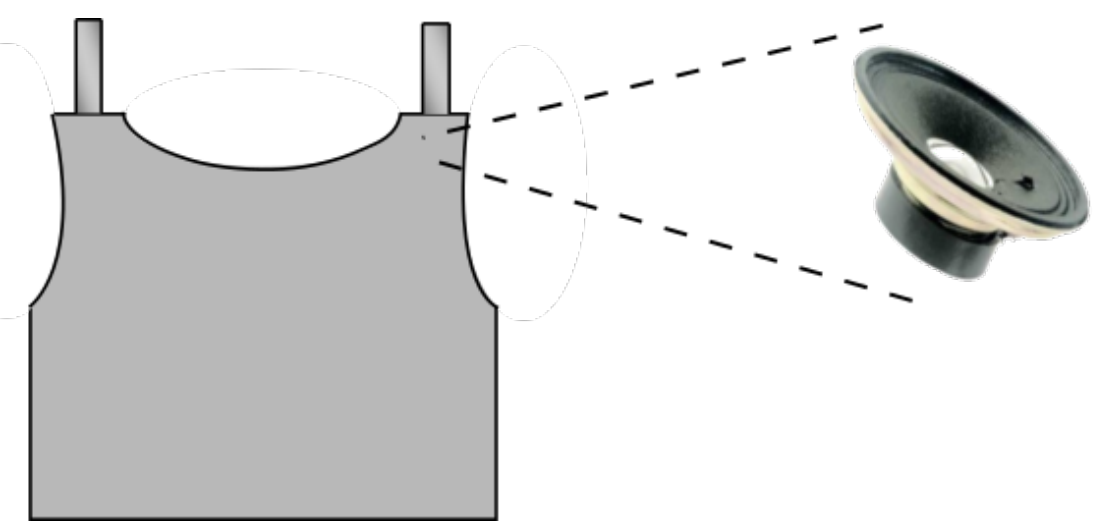

Figure 7. Location of the speaker for the kissing affection gesture.

\section{Evaluation of the Redesigned AffectiVest}

The objective of this evaluation was to observe the behavior of the grandparents and their grandchildren while using the prototype during their communication, to determine if it enriches the communication process. For this, a formative evaluation was carried out in terms of usability, usefulness and user experience of the prototype. In addition, a couple of sensors were used to register possible physiological changes in the participants when receiving the affection gestures from the prototype. These data will be used to carry out a psychophysiological evaluation, which for reasons of scope of the present work, will be analyzed and reported as future work.

\subsection{Participants}

A total of 10 voluntary grandparent-grandchild couples participated in the evaluation, 10 older adults with an average age of 69.8 years (SD 10.96) and 10 children with an average age of 8.2 years (SD 1.72). An inclusion criterion for the participants was that they should have a grandparent-grandchild relationship. Each participant was rewarded with a small treat as a thank you for participating in the experiment.

\subsection{Procedure}

On-entry: Informative meeting, the objective of the evaluation and the operation of the redesigned prototype was explained to the participants. Previous screening, to know the state of their soft neurological signs, and ensure that they did not present any problem that prevented them from receiving the affection gestures correctly from the prototype. Evaluation of soft neurological signs, the Infant Neuropsychological Assessment (ENI) was applied [14], focused on evaluating sight, hearing and touch (senses necessary for the reception of information from the prototype). To discard symptoms of depression in the elderly, the geriatric depression scale, Yasevage Test [15] was applied. None of the participants presented any inconvenience, therefore none of them was ruled out.

Treatment: Stage 1. Communicating and wearing the AffectiVest. Each pair of participants was placed in different laboratory rooms and participants wore the AffectiVests to be able to receive the affection gestures (see Figure 8). A plethysmograph (to record the frequency and amplitude of breathing) and an electromyograph (to record voltage changes in the muscles of the abdomen) were also placed on the participants to obtain data for a later psychophysiological evaluation. Then, they were explained that in the first stage they would communicate with each other through a video call using a computer, and that they would not use the prototype even if they were wearing it, the idea was that they would wear it in this stage only to get used to it. The computers were ready to initiate communication between the participants and the duration of the video call was approximately $3 \mathrm{~min}$ for each pair, time enough to address the communication and move on to the next stage. 


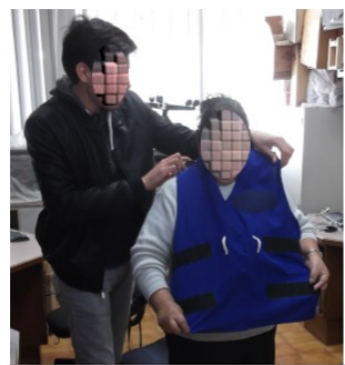

(a)

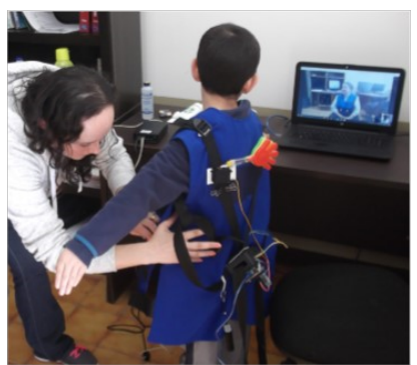

(b)

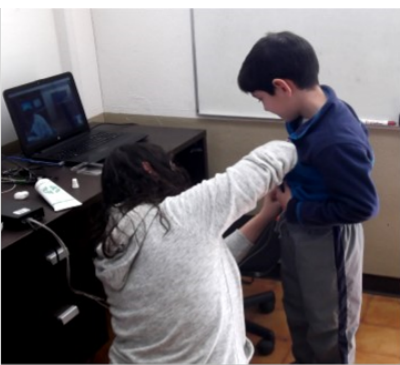

(c)

Figure 8. Participants putting on the AffectiVests: (a) Grandmother, (b) grandchild and (c) Putting the plethysmograph and electromyograph on the grandchildren.

Stage 2. Communicate and send affective gestures to self. The objective of this stage was for the participants to become familiar with the use of the prototype as a tool to enrich the communication between grandparents and grandchildren. For this, each participant was given the sender application to interact with it. The participants would feel the affection gestures created by their own interaction, sending themselves the four available affection gestures. Participants freely explored the use of the sender application; that is, they selected from the smartphone the affection gesture they would like to feel as well as the number of times to reproduce it.

Stage 3. Communicating and sending affective gestures to the other. Participants exchanged the sender applications so that they could send affection gestures to the other, and the other could receive them. Participants continued communicating using the video call and were given approximately three minutes to use the application freely (see Figure 9). After that time, the participant not wearing the physiological sensors was given a short story and instructed to read it to his/her relative. The story included several instances of the four affection gestures; and participants were instructed so that each time an affection gesture appeared in the short story, they select it in the sender application to be sent to, and received by, his/her relative. Each affection gesture appeared three times in the story, to ensure that the participant send them all. On finishing reading the story, the video call finished, and the AffectiVests and physiological sensors were removed.

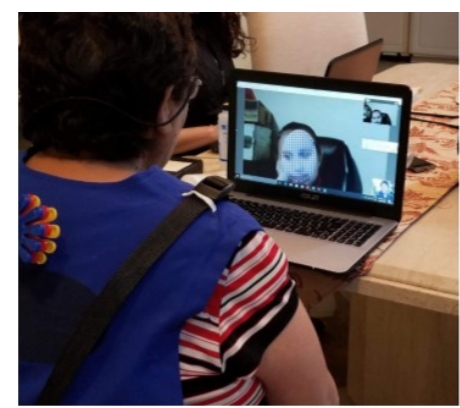

(a)

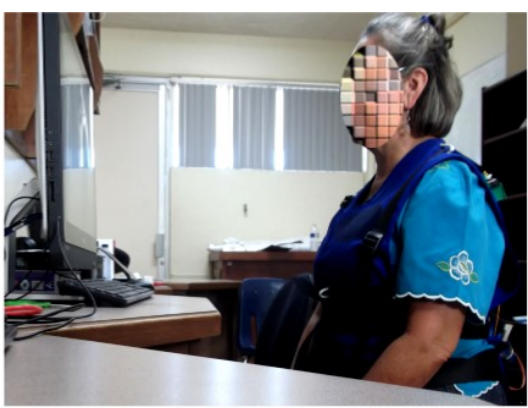

(b)

Figure 9. (a,b) Participants in stage 3.

On-exit: Software Usability Scale (SUS-[16]), the SUS questionnaire was applied to obtain the participants' perception regarding the usability of the prototype with a 5-point Likert scale; Technology Acceptance Model (TAM-[17]), the TAM questionnaire was also applied to obtain their perception regarding the usefulness and ease of use of the prototype with a 7-point Likert scale; and Fidelity of affection gestures questionnaire, additional questions were included to obtain the participants' perception regarding the fidelity of the affection gestures received through the AffectiVest with a 5-point Likert scale. On average, the duration of the experiment was $50 \mathrm{~min}$ per couple, ranging from the previous screening to the application of the on-exit questionnaires. 


\section{Results and Discussion}

\subsection{Results of the Fidelity of Affection Gestures questionnaire}

The results of the fidelity of affection gestures questionnaire are summarized in Table 1. It can be observed that the best-qualified affection gesture was that of the Tickling on the abdomen gesture, which was perceived by $90 \%$ of the participants as Very real (14/20) or Real (4/20) and only $10 \%$ perceived it as Real enough (2/20). The second best rated was the Tapping on the shoulder gesture, which was perceived by $85 \%$ of the participants as Very real $(12 / 20)$ or Real $(5 / 20)$ and by $5 \%$ as Real enough $(1 / 20)$; however $10 \%$ of the participants perceived it as Slightly real $(2 / 10)$. The third best perceived affection gesture was the Kissing gesture, which was perceived by $70 \%$ of the participants as Very real $(9 / 20)$ or Real $(5 / 20)$, and by $30 \%$ as Real enough $(6 / 20)$. The worse qualified affection gesture was the Hugging gesture, which was perceived only by $45 \%$ of the participants as Very real (7/20) or Real (2/20), and by 35\% as Real enough (7/20), even more, $20 \%$ of the participants perceived it as Slightly real $(2 / 10)$.

Table 1. Results of the Fidelity of Affection Gestures questionnaire.

\begin{tabular}{ccccc}
\hline & Hugging & Kissing & Tickling on the Abdomen & Tapping on the Shoulder \\
\hline Unreal & $0 \%$ & $0 \%$ & $0 \%$ & $0 \%$ \\
Slightly real & $20 \%$ & $0 \%$ & $0 \%$ & $10 \%$ \\
Real enough & $35 \%$ & $30 \%$ & $10 \%$ & $5 \%$ \\
Real & $10 \%$ & $25 \%$ & $20 \%$ & $25 \%$ \\
Very real & $35 \%$ & $45 \%$ & $70 \%$ & $60 \%$ \\
\hline
\end{tabular}

\subsection{Results of the SUS Questionnaire}

The results of the SUS questionnaire regarding the usability of the prototype yielded a score of $84.37 / 100$ with a standard deviation of 1.12. According to the usability scale presented by [18], the usability perceived by the participants places the prototype within an acceptable range and with an A grade, that is, with a rating of Good to Excellent. Likewise, it is located above the third quartile indicating that more than $75 \%$ of the participants (15/20) answered "Completely Agree" that the prototype is very usable (see Figure 10), using a Likert scale of 0 (Completely Disagree) to 4 (Completely Agree).

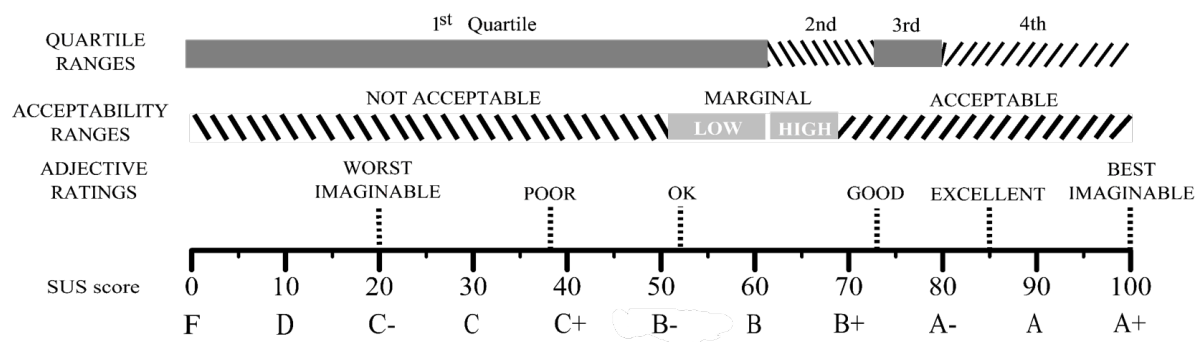

Figure 10. Comparison of the Software Usability Scale (SUS) score by quartiles, acceptability and adjective qualification. Figure adapted from [18].

To obtain more information regarding the answers of the participants, in Figure 11 the results are presented using a curved rating scale [19], where it can be seen that $70 \%$ of the participants (14/20) perceived a high usability obtaining an adjective rating between "A+" "Best imaginable" and " $\mathrm{A}$ " (Excellent). On the other hand, $10 \%$ of the participants $(2 / 20)$ perceived that the prototype had a sufficient usability margin, qualifying it between "B+" (Good) and "B-" (Ok). Something worrisome was that to $15 \%$ of the subjects $(3 / 20)$ the usability seemed not acceptable, qualifying it as " $\mathrm{C}^{\prime \prime}$ (Poor) and even $5 \%$ of them $(1 / 20)$ rated it as " $F$ " (Worst imaginable). A possible explanation for this last point is presented in the following subsection. 


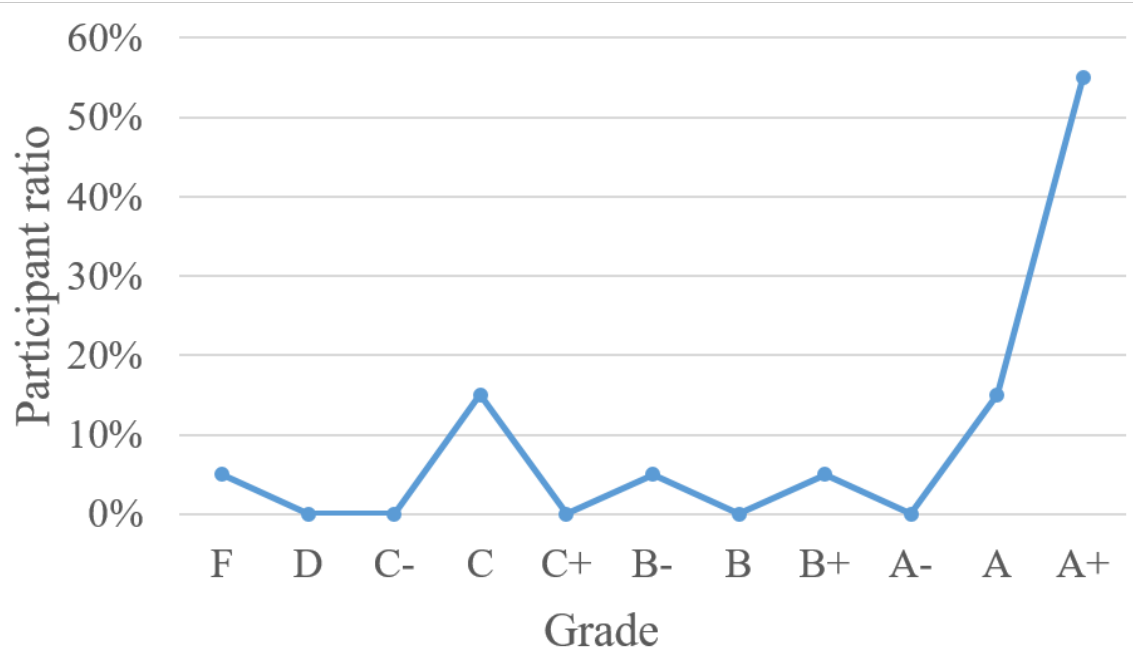

Figure 11. Curved classification scale of the score obtained from the SUS questionnaire.

\subsection{Results of the TAM Questionnaire}

The results of the TAM questionnaire are separated in terms of perceived usefulness and ease of use. Table 2 shows the responses of perceived usefulness; as well as its measures of central tendency and standard deviation. These results show that most of the participants answered "Extremely Agree" regarding the usefulness they perceived from the prototype. On average the participants' answers are located at 5.6 (Strongly Agree to Extremely Agree) deviating 1.12 units from the scale. Only one participant answered "Extremely Disagree" regarding the usefulness of the prototype. For the rest, $75 \%$ of the scores awarded are located in the high to highest values of the scale (Slightly Agree to Extremely Agree).

Table 2. Participants' perceived usefulness.

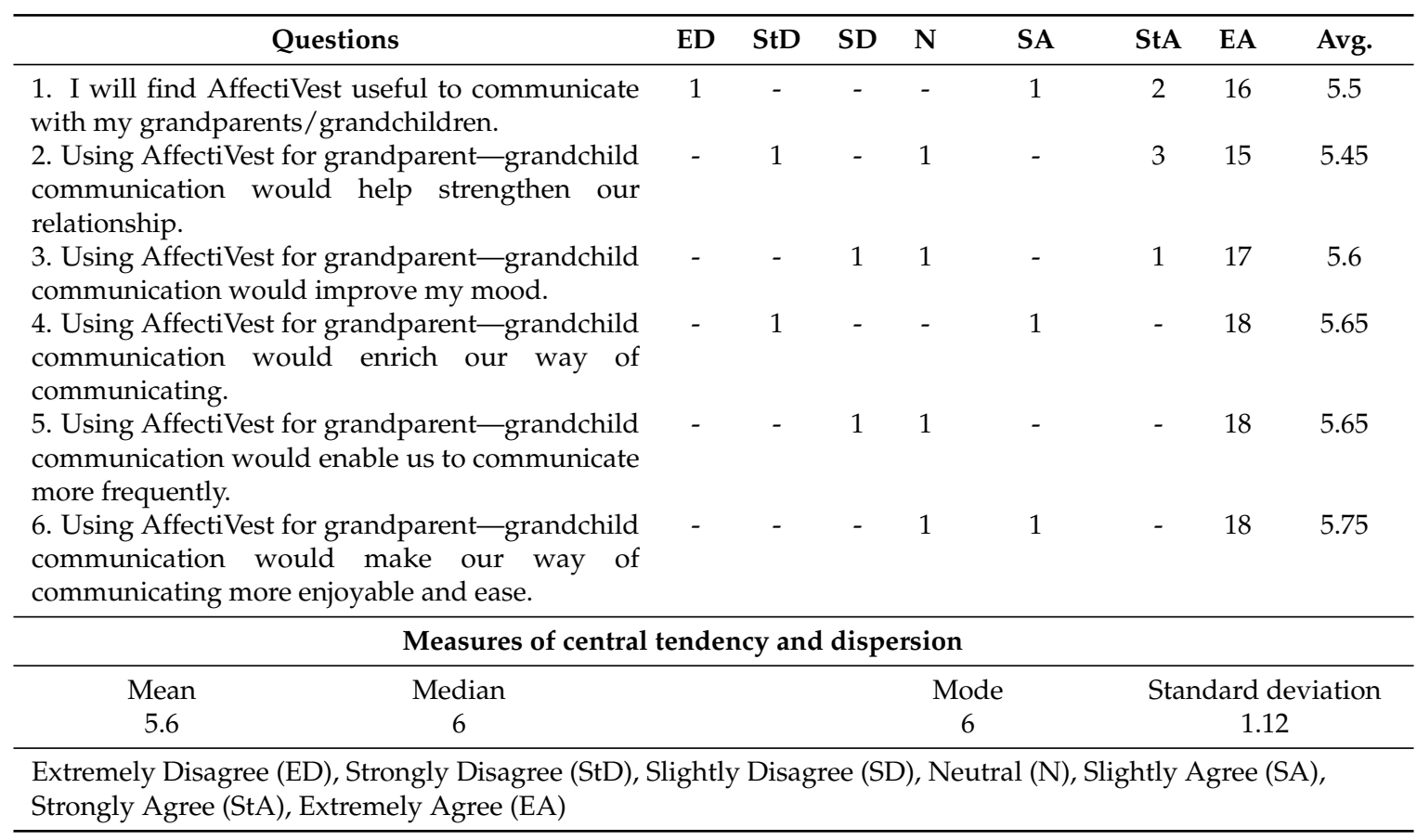

Table 3 shows the perceived ease of use responses; as well as its measures of central tendency and standard deviation. Similar to the usefulness case, these results show that most of the participants answered "Extremely Agree" regarding the ease of use perceived in the prototype. On average, 
participants' answers stand at 5.48 (Strongly Agree to Extremely Agree), deviating 1.15 units from the scale. As for usefulness, only one participant gave a rating of "Extremely Disagree". For the rest, $65 \%$ of the scores awarded are located in the high to highest values of the scale (Slightly Agree to Extremely Agree).

Table 3. Participants' perceived ease of use.

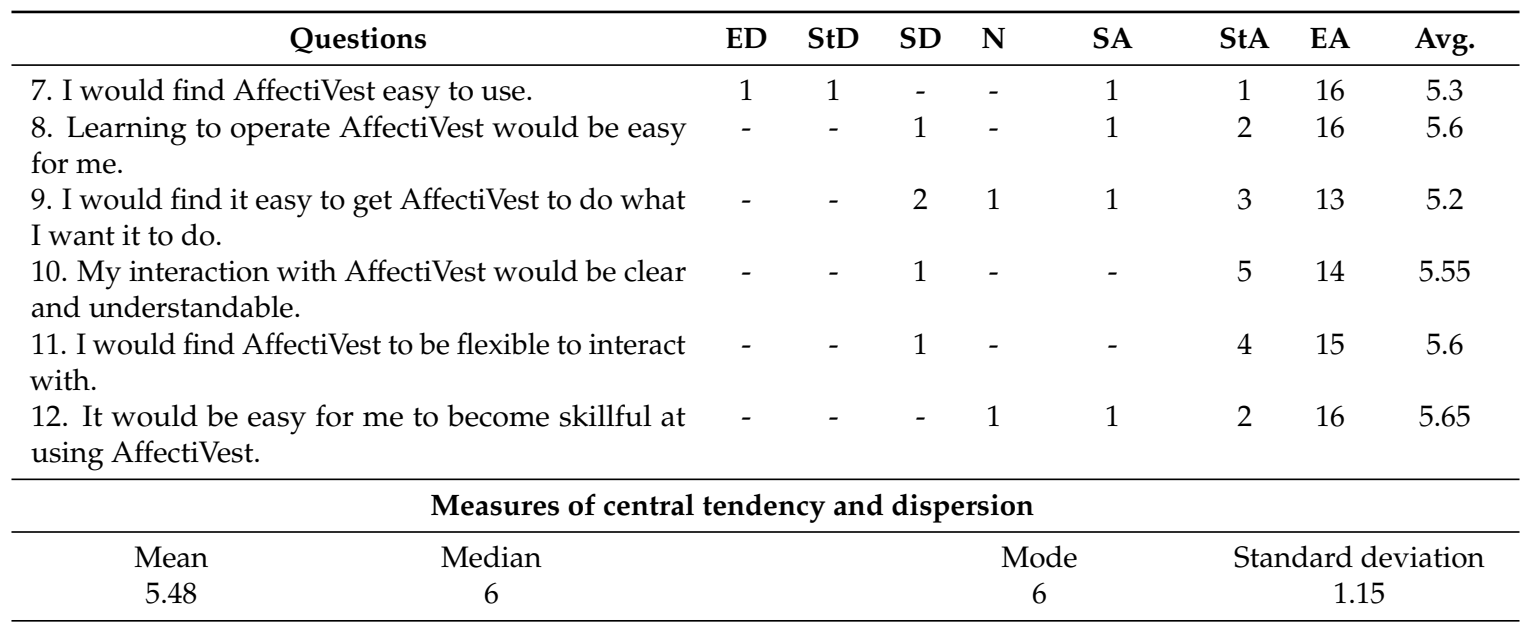

Extremely Disagree (ED), Strongly Disagree (StD), Slightly Disagree (SD), Neutral (N), Slightly Agree (SA), Strongly Agree (StA), Extremely Agree (EA)

One possible explanation for the rating of "Extremely Disagree" for both perceived usefulness and ease of use by one participant, is that the participant (older adult) who gave this rating had a slight problem in the back, for this reason it was not possible to adequately adjust AffectiVest to his/her torso, and after the evaluation $\mathrm{s} / \mathrm{he}$ indicated that due to this $\mathrm{s} /$ he had not managed to perceive some of the stimuli of the affection gestures.

\section{Conclusions and Future Work}

The results of the fidelity questionnaire of the affection gestures indicate that it can be determined that the redesign of the mechanisms to represent the stimuli of each affection gesture in the AffectiVest wearable device was adequate and intuitive for the participants, evoking all the affection gestures as Real enough to Very real to at least $80 \%$ of them. Particularly, in the second version of AffectiVest the redesigned Tickling on the abdomen and Tapping on the shoulder affection gestures were perceived by $90 \%$ and $85 \%$ of the participants as Real or Very real, respectively; in comparison to the original version where these two affection gestures were the least well perceived. On the other hand, the Hugging affection gesture, which in the first version of the prototype was the best evoked gesture in the participants; in the second version it was the least best evoked gesture, being perceived by $20 \%$ of the participants as Slightly real. Although the redesign of this affection gesture improved the participants' perception by eliminating the source of noise of the first version, there is still a large opportunity to improve its design. Further, although the redesigned AffectiVest AWC prototype presented high usability and usefulness according to the results of the SUS and TAM questionnaires, the participants suggested some improvements regarding the wearable device. They particularly suggested to: i) modify the size of the vest so that it could fit more adequately to the proportions of smaller users and allow us to perceive the stimuli corresponding to each affection gesture in the intended zones (e.g. abdomen and shoulder) and ii) improve its design so that the participants can perform their daily activities without interfering with them. As future work, firstly in order to improve our understanding of the fidelity of the affection gestures and to contrast the results obtained in this questionnaire-based evaluation regarding user experience, usability and ease of use, the data captured with the physiological sensors will be analyzed and used to determine if and to what extent there 
are changes in the respiratory rate and abdominal muscle tone of the participants when receiving the stimuli of the affection gestures; in addition, we will continue improving the device by taking into consideration the recommendations of the participants.

Author Contributions: Conceptualization, F.B.M., A.L.M.; Methodology, F.B.M, V.M.-K., C.R.-F., A.L.M.; Software, F.B.M.; Validation, F.B.M, V.M.-K., C.R.-F., A.L.M.; Formal analysis, F.B.M, V.M.-K., C.R.-F., A.L.M.; Investigation, F.B.M, V.M.-K., C.R.-F., A.L.M.; Resources, F.B.M, V.M.-K., A.L.M.;Writing-Original Draft Preparation, F.B.M, V.M.-K., C.R.-F., A.L.M.; Writing-Review \& Editing, F.B.M, V.M.-K., C.R.-F., A.L.M.; Visualization, F.B.M, V.M.-K.; Supervision, A.L.M.; Project Administration, F.B.M, V.M.-K., A.L.M.; Funding Acquisition, V.M.-K., A.L.M.

Funding: This research was partially funded by UABC under grant 0239 of the 21a Convocatoria Interna de Apoyo a Proyectos de Investigación and under grant 0212 of the Convocatoria de Proyectos de Servicio Social 2019.

Acknowledgments: The authors would like to thank the subjects for their participation in the evaluation studies, Eloisa García-Canseco for her following the work of the first author, Xavier Chénot, Jorge A. I. Hernández and Josué González-Martínez for their work on the redesign of the hardware and software components of AffectiVest, and the Consejo Nacional de Ciencia y Tecnología of Mexico for scholarship number 446372 provided to the first author.

Conflicts of Interest: The authors declare no conflict of interest.

\section{References}

1. Wickramage, K.; Vearey, J.; Zwi, A.B.; Robinson, C.; Knipper, M. Migration and health: a global public health research priority. BMC Public Health 2018, 18, 987.

2. Kornhaber, A.; Woodward, K.L. Grandparents, Grandchildren: The Vital Connection; Transaction Publishers: New Brunswick, NJ, USA, 1981.

3. Kornhaber, A. Contemporary Grandparenting; Sage: Thousand Oaks, CA, USA, 1996.

4. Ross, N.; Hill, M.; Sweeting, H.; Cunningham-Burley, S. Grandparents and teen grandchildren: Exploring intergenerational relationships. Technical report, CRFR, 2005.

5. Becker, O.A.; Steinbach, A. Relations between Grandparents and Grandchildren in the Context of the Family System. Comp. Popul. Stud. 2012, 37. doi:10.4232/10.CPoS-2012-06en.

6. Ballagas, R.; Kaye, J.; Ames, M.; Go, J.; Raffle, H. Family communication: phone conversations with children. In Proceedings of the 8th ACM International Conference on Interaction Design and Children, Como, Italy, 3-5 June 2009; pp. 321-324.

7. Forghani, A.; Neustaedter, C.; Schiphorst, T. Investigating the communication patterns of distance-separated grandparents and grandchildren. In Proceedings of the CHI'13 ACM Extended Abstracts on Human Factors in Computing Systems, Paris, France, 27 April-2 May 2013; pp. 67-72.

8. Picard, R.W.; Healey, J. Affective wearables. Pers. Technol. 1997, 1, 231-240.

9. Mueller, F.; Vetere, F.; Gibbs, M.R.; Kjeldskov, J.; Pedell, S.; Howard, S. Hug over a distance. In Proceedings of the CHI'05 extended abstracts on Human factors in computing systems, Portland, OR, USA, 2-7 April 2005; pp. 1673-1676.

10. Teh, J.K.; Tsai, Z.; Koh, J.T.; Cheok, A.D. Mobile implementation and user evaluation of the Huggy Pajama system. In Proceedings of the 2012 IEEE Haptics Symposium (HAPTICS), Vancouver, BC, Canada, 4-7 March 2012; pp. 471-478.

11. Rosella, F.; Genz, R. Wearable Haptic Telecommunication Device and System. US Patent App. 11/515,690, 22 March 2007.

12. Cabibihan, J.J.; Chauhan, S.S. Physiological responses to affective tele-touch during induced emotional stimuli. IEEE Trans. Affect. Comput. 2015, 8, 108-118.

13. Montañez, F.B.; Morán, A.L.; Meza-Kubo, V.; García-Canseco, E. Evaluation of an Affective Wearable Tool for the Transmission of Affection Gestures Between Geographically Separated Loved Ones. In Proceedings of the 2017 International Conference on Ubiquitous Computing and Ambient Intelligence (UCAMI), Philadelphia, PA, USA, 7-10 November, 2017; pp. 808-813.

14. Matute, E.; Rosselli, M.; Ardila, A.; Ostrosky-Solís, F. Evaluación Neuropsicológica Infantil; Manual Moderno: Mexico, 2007.

15. Yesavage, J.A.; Brink, T.L.; Rose, T.L.; Lum, O.; Huang, V.; Adey, M.; Leirer, V.O. Development and validation of a geriatric depression screening scale: a preliminary report. J. Psychiatr. Res. 1982, 17, 37-49.

16. Brooke, J. SUS-A quick and dirty usability scale. Usability Eval. Ind. 1996, 189, 4-7. 
17. Davis, F.D. Perceived usefulness, perceived ease of use, and user acceptance of information technology. MIS Q. 1989, 13, 319-340.

18. Bangor, A.; Kortum, P.T.; Miller, J.T. An empirical evaluation of the system usability scale. Int. J. Hum. Comput. Interact. 2008, 24, 574-594.

19. Sauro, J.; Lewis, J.R. Quantifying the User Experience: Practical Statistics for User Research; Morgan Kaufmann: San Francisco, CA, USA, 2016.

(C) 2019 by the authors. Licensee MDPI, Basel, Switzerland. This article is an open access article distributed under the terms and conditions of the Creative Commons Attribution (CC BY) license (http://creativecommons.org/licenses/by/4.0/). 